\title{
A message from the new Editor-in-Chief of the International Journal of Gynecological Cancer
}

\author{
Pedro T Ramirez
}

Department of Gynecologic Oncology, The University of Texas MD Anderson Cancer Center, Houston, Texas, USA

\section{Correspondence to} Dr Pedro T Ramirez, Department of Gynecologic Oncology, The University of Texas MD Anderson Cancer Center, Houston, TX 77030, USA; peramire@mdanderson. org

Received 21 November 2018 Accepted 21 November 2018 Published Online First 19 December 2018

Check for updates (C) IGCS and ESGO 2019. No commercial re-use. See rights and permissions. Published by BMJ.

To cite: Ramirez PT. Int J Gynecol Cancer 2019;29:1-2.
I am truly honored to have been selected as the new Editor-in-Chief of the International Journal of Gynecological Cancer. I am also very proud to be working in tandem with an outstanding team of Associate Editors and members of the Editorial Board. The latter have been selected as a balanced global representation of the leadership in our field. This is an editorial team that is fully engaged and committed to the success of this outstanding journal.

In this new era of the International Journal of Gynecological Cancer there are a number of changes that I would like to highlight to the journal's readers, as we are confident such changes will appeal to a wide range of academic and clinical interests. First, our journal has a new cover that has been collectively designed by our leadership team that aims to represent the global network of our community of gynecologic oncologists. We recognize the importance of ensuring that our journal represents the work and research being conducted in all regions of the world, and at the same time also highlights key issues critical to patients not only in developed countries but also in low-resource countries. Second, the journal will feature Original Articles that showcase important and innovative work within the field of gynecologic oncology, and in each journal issue the Editor-in-Chief and Associate Editors will select a Lead Article that they feel contains research of a particularly high caliber and/ or scientific impact. This article will have an accompanying Lead Article Editorial, as well as a podcast by the authors outlining the relevance of their work and its applicability to current clinical practice. Third, the journal will feature a new section entitled Clinical Trials. In this segment we will highlight the protocol details of prospective multi-institutional trials that are currently ongoing or due to begin patient accrual. The aim of Clinical Trials is to increase readers' awareness of the specifics of such protocols and provide them with opportunities for collaboration. Fourth, each issue will include at least one commissioned Review Article written by well-recognized figures in our field, so that journal readers can be apprised about the most up-todate literature on relevant and current topics. Fifth, another very exciting new feature of the journal will be Case Studies. These comprise cases presented in the format of a multi-disciplinary conference, where the presenters will provide case details with input from pathologists, radiologists, and/ or radiation oncologists, with a skilled discussant answering key questions on management decision and counseling for the patient concerned. Sixth, we will have a feature entitled Corners of the World. This will be a short synopsis of the experiences of members of our global community of gynecologic oncologists describing their work in rural and disadvantaged regions of the world, with an emphasis on how such work impacts on patient care. There is tremendously valuable work being done by many gynecologic oncologists that often goes unrecognized and we aim to provide a forum to showcase its impact. Seventh, we will feature a series of podcasts and videos that will be freely available to all users. The podcasts will be published on a weekly basis and they will feature interviews with key leaders in our field discussing topics pertinent to their area of expertise. The videos will aim to demonstrate unique surgical or diagnostic approaches in the management of patients with gynecologic cancers. Lastly, we have implemented a targeted social media campaign that will facilitate rapid and easy access to upcoming articles in the journal as well as a forum for readers to participate in monthly journal clubs. In summary, we want our journal to be your primary source of information in gynecologic oncology.

As Editor-in-Chief, I recognize the value authors place on high-quality and unbiased peer review conducted in a timely manner. In addition, we value the importance of rapid publication, and so to that end we have structured our editorial team to comprise Associate Editors, a Social Media Editor, and a Video Editor so we are able to expedite the processing of submitted manuscripts. I have instructed all those involved with the journal in an effort to provide the highest standard of manuscript review, editing, and publishing. We have implemented rigorous peer review criteria, and this will be reflected in the quality of published articles. We also want to encourage all those who are interested 


\section{Editorial}

in being part of this energetic and enthusiastic team to contact us, as we will welcome your contribution. We invite colleagues working in related disciplines such as, but not limited to, medical oncology, pathology, radiology, and radiation oncology to consider our journal as an appropriate medium for the publication of your own high-quality research.

I am extremely excited to be embarking on this very important role and wish to express my gratitude to the leadership and the selection committee of the International Gynecologic Cancer Society and the European Society of Gynecologic Oncology for electing me as the new Editor-in-Chief of this influential journal. I would also like to thank Dr Uzi Beller and his editorial team for all the years that they have devoted to the growth and development of the journal, and also wish to thank Dr Beller for his efforts in ensuring a smooth transition. In addition, I would like to thank our commercial publisher, BMJ, and their devoted team, for their outstanding work, guidance, and encouragement with this new venture. Equally, I would like to thank our Managing Editor, Lindsey Struckmeyer, and J\&J Editorial for their continued and substantial assistance with the successful launch of the journal. Thank you all once again for your amazing support and continued efforts aimed at ensuring that the International Journal of Gynecological Cancer is recognized as the leading journal in its field.

Contributors PTR is the sole author of the Editorial.

Funding The authors have not declared a specific grant for this research from any funding agency in the public, commercial or not-for-profit sectors.

Competing interests None declared.

Patient consent Not required.

Provenance and peer review Not commissioned; internally peer reviewed. 